\title{
Related subjects
} Pulmonary hypertension

\section{[gw22-e0008] EVALUATION OF TWO-DIMENSIONAL STRAIN ECHOCARDIOGRAPHY IN OUANTIFYING RIGHT VENTRICULAR FUNCTION IN PATIENTS WITH PULMONARY HYPERTENSION}

Liu Yong, ${ }^{1}$ Pan Lei, ${ }^{1}$ Zhang Zhenning, ${ }^{1}$ Shi Zhihua, ${ }^{1}$ Wang Yong, ${ }^{1}$ Jing Zhicheng ${ }^{2}{ }^{1}$ Beijing

Shijitan Hospital, Beijing, China; ${ }^{2}$ Shanghai Thoracic Hospital, Xuhui, China

10.1136/heartjnl-2011-300867.666

Objective To evaluate RV longitudinal function in pulmonary hypertension (PH) patients by two-dimensional strain (2DS).

Methods Forty one patients with $\mathrm{PH}$ were prospectively studied along with 40 control patients. RV 2DS and right heart catheterisation were performed, peak systolic longitudinal strain $(\varepsilon)$ and strain rate (SRs) obtained from basal to apical RV free wall were calculated. RV deformation indices were compared with values of conventional echocardiography and right heart catheterisation with the use of linear regression.

Results (1) $\varepsilon$, SRs of all RV free wall segments in PH group were significantly lower than those in the control group ( $p<0.05) ; \varepsilon$, SRs of the middle and apical segment in $\mathrm{PH}$ group were significantly lower than basal segment $(p<0.05)$. (2) SRs of RV apical and middle segment in $\mathrm{PH}$ group correlated well with RV size and diameter. Also, $\varepsilon$ of RV apical segment correlated well with conventional echocardiography measurement of RV size and systolic function. (3) $\varepsilon$, SRs of the apical segment in PH group correlated well with right heart catheterisation measurement of pulmonary artery pressure, pulmonary vascular resistance and stroke volume.

Conclusion The right ventricular longitudinal systolic function can be evaluated accurately and objectively by two-dimensional strain echocardiography. 